\title{
Determination of Extratropical Tropopause Height in an Idealized Gray Radiation Model
}

\author{
PABLO ZURITA-GOTOR \\ Universidad Complutense, and Instituto de Geociencias UCM, CSIC, Madrid, Spain \\ GEOFFREY K. VALLIS \\ Princeton University, Princeton, New Jersey
}

(Manuscript received 23 July 2012, in final form 3 February 2013)

\begin{abstract}
This paper investigates the mechanisms that determine the extratropical tropopause height, extending previous results with a Newtonian cooling model. A primitive equation model forced by a meridional gradient of incoming solar radiation, with the outgoing infrared radiation calculated using a simple gray radiation scheme, is now used. The tropopause is defined as the top of the boundary layer over which dynamical heat transport moves the temperature away from radiative equilibrium, and its height is estimated from the isentropic mass flux. Depending on parameters, this tropopause may or may not be associated with a sharp stratification change, and it may or may not be possible to define a thermal tropopause. The mass flux and thermal tropopause display similar sensitivity to external parameters when the latter can be defined; this is a sensitivity in good agreement with predictions by a radiative constraint. In some contrast to the Newtonian model, the radiative constraint is now quite effective in preventing adjustment to marginal criticality with realistic parameters.

The meridional structure of the thermal tropopause displays a jump in height at the jet latitude, which appears to be due to the formation of a mixing barrier at the jet maximum when baroclinicity has a finite vertical scale. As meridional potential vorticity mixing is inhibited across the jet, a discontinuity is created between weakly stratified air on its warm side and strongly stratified air on its cool side. The meridional stratification contrast is created by adiabatic cooling and warming by the residual circulation, as this circulation must be deflected vertically to avoid the mixing barrier at the jet maximum.
\end{abstract}

\section{Introduction}

The determination of the tropopause height is one of the fundamental problems in general circulation theory, with important implications for climate and climate change. Although we have a qualitative understanding of the processes that give rise to the tropopause, we still lack a simple theory to predict its height. Given the complex and multidisciplinary nature of the tropopause determination problem it is perhaps naïve to think that such a simple theory might even exist. Dynamical, diabatic, and chemical processes are all thought to play a role, and indeed there are many definitions of the tropopause. Although they are all quantitatively similar they differ in

Corresponding author address: Pablo Zurita-Gotor, Departamento de Geofísica y Meteorología, Universidad Complutense, Facultad de Ciencias Físicas, Avenida Complutense, Madrid 28040, Spain.

E-mail:pzurita@alum.mit.edu details and even in concept (World Meteorological Organization 1957; Hoerling et al. 1991; Pan et al. 2004; Kunz et al. 2011). For many chemical species a smooth transition is observed between their characteristic tropospheric and stratospheric values, motivating the interpretation of the tropopause as a finite transition layer rather than as a sharp boundary [see Fueglistaler et al. (2009) and Gettelman et al. (2011) for recent reviews on the tropical and extratropical tropopauses]. Yet the thermal tropopause at any given time is often very sharp-sharper than the smeared Eulerian time average would indicate (Birner 2006). Given all this complexity, it makes sense to approach this problem in the simpler context of an idealized model, using simplified dynamics and heating and ignoring chemical feedbacks. This is our goal with this paper, which extends previous results by Zurita-Gotor and Vallis (2011, hereafter ZGV).

At a fundamental level, the tropopause may be regarded as the depth of the boundary layer (the troposphere) 
over which the dynamical motions redistribute the heat received at the surface. Held (1982) proposed a minimal conceptual framework for understanding the determination of this depth, consisting of a stratosphere in strict radiative equilibrium matching with continuous temperature the underlying troposphere-an active layer whose mean thermal gradients are shaped by the dynamical transport. In particular, the vertical heat transport may be thought of as adjusting the tropospheric stratification to some characteristic value $\tilde{\theta}_{z}$, stabilizing the lower troposphere and destabilizing the upper troposphere and leading to a stratification discontinuity at the tropopause. Held noted that in this minimal representation the tropopause height $H$ and the tropospheric stratification $\tilde{\theta}_{z}$ fully determine the net column heating so that there must be a relation between them: $H=f\left(\tilde{\theta}_{z}\right)$ when this heating is known (for instance, if we uncouple the horizontal and vertical transport problems). Held called this relation the radiative constraint. As ZGV note, the radiative constraint is a statement of energy conservation and must always be satisfied. The constraint implies that he vertical thermal structure cannot be fully arbitrary because the net column heating must balance the meridional divergence. However, the idealized construction that the stratosphere is in radiative equilibrium and the tropospheric stratification is uniform is only an approximation, and the radiative constraint need not provide an effective constraint on tropopause height; that is, small changes in the heating might lead to large changes in tropopause height.

To close the problem, an additional dynamical constraint between $H$ and $\tilde{\theta}_{z}$ is needed. One plausible possibility is baroclinic adjustment-a geometric constraint on the isentropic slope based on neutrality to baroclinic instability (Stone 1978; Held 1982); see Zurita-Gotor and Lindzen (2007) for a review. [A different argument for a closely related constraint was given by Schneider (2004) and Schneider and Walker (2006) as an adjustment to marginal criticality, using different assumptions not requiring neutrality.] Another possibility is to suppose some form of turbulent diffusion of heat. In general, turbulent diffusion and an adjustment to marginal criticality can both hold only if the turbulent diffusivity has a particular form; otherwise, in the presence of an effective radiative constraint, the system is overconstrained. Note too that adjustment arguments suggest that as parameters are changed in the vicinity of marginal criticality then fluxes should vary very rapidly in order to keep the state marginally supercritical, whereas turbulent diffusion is generally associated with a more continuous dependence on parameters.

The evidence for adjustment is mixed. Schneider (2004) and Schneider and Walker (2006) do find strong evidence of adjustment in an idealized model forced by Newtonian cooling, but no such evidence is found in models with more realistic heating (Thuburn and Craig 1997; Frierson et al. 2006). In models forced using Newtonian cooling, marginal criticality adjustment is observed in some regimes but not in others depending on the structure of the heating or the fluid properties (Zurita-Gotor 2008; Jansen and Ferrari 2012). Turbulent diffusive closures (and not marginal criticality) seem effective in atmospheric models bounded by a top rigid lid (Zurita-Gotor and Vallis 2009), although actually producing a theory for the diffusivity is extremely difficult for a continuous fluid.

There are circumstances in which it appears that both dynamical constraints might be effective. Thus, $\mathrm{ZGV}$ found that adjustment to marginal criticality is compatible with turbulent diffusion closures when the tropopause height is essentially determined by the dynamics and largely unconstrained by the heating. The radiative constraint still formally applies, of course, but does not constrain the height of the tropopause and marginal criticality appears to hold well. The freedom in the height of the tropopause allows the turbulent diffusivity sufficient freedom so that it, too, can hold in the sense that the fluxes vary smoothly with parameters (it is not possible to test the diffusive theory more thoroughly in the absence of a quantitative closure). If, on the other hand, the thermodynamics effectively control the tropopause height, then the system cannot simultaneously satisfy the independent dynamical constraints implied by marginal criticality and the turbulent diffusion closure unless additional degrees of freedom are allowed (see section 1 of $\mathrm{ZGV}$ for a more detailed discussion of these points).

Aside from the evident difficulty in understanding the circumstances in which the dynamical constraints hold, we may also wonder, given its severe idealizations, if the radiative constraint is meaningful in Earth's atmosphere. For example, the stratosphere departs significantly from radiative equilibrium (Andrews et al. 1987), meaning that the actual radiative constraint (i.e., energy balance) involves the structure of the atmosphere above the tropopause. Given this, stratospheric dynamical heating can be expected to be important for tropopause height, as in fact found by Thuburn and Craig (2000). Further, the finite temperature change across the shallow inversion layer observed right above the tropopause (Birner 2006) introduces an additional degree of freedom in the vertical thermal structure even when the overlying stratosphere is in radiative equilibrium. Finally, the assumption of uniform tropospheric stratification is also clearly an idealization. Yet despite all these shortcomings the radiative constraint seems to 
work well in a comprehensive GCM when parameters are varied (Thuburn and Craig 1997).

We (ZGV) have recently proposed that the extent to which the radiative constraint is satisfied or not may depend on the strength of the vertical heat transport/ stratification adjustment in the troposphere. When this transport is large, corrections to the heating arising from the violation of the idealized assumptions in the radiative constraint should be small compared to the divergence of the dynamical transport, and the idealized construction may be expected to work. In contrast, for small vertical heat transport these corrections may be as important as the dynamical heating itself, rendering the idealized construction inaccurate and its predictions ineffectual. The magnitude of the required vertical transport to produce any given tropospheric stratification $\tilde{\theta}_{z}$ is a property of the radiative constraint, and can be estimated prior to any simulation regardless of whether that constraint is satisfied or not.

To quantify this transport and assess the reliability of the radiative constraint, in $\mathrm{ZGV}$ we proposed to use the sharpness of the stratification jump at the tropopause, indicative of the impact of dynamical heating for upper-troposphere stratification. We refer to this as the tightness of the radiative constraint. Another important property of the radiative constraint introduced in $\mathrm{ZGV}$ is its steepness, which measures the flexibility of tropopause height when stratification is varied. Depending on the characteristics of the heating, the radiative constraint may effectively impose a rigid lid and not let the tropopause move much, or it may be very flexible and effect little control on tropopause height. In the first case the flow can be supercritical and an inverse cascade might be expected (Zurita-Gotor and Vallis 2009), whereas adjustment to marginal criticality seems more likely in the second case when the tropopause height is less constrained by the heating. ZGV also shows that tightness and steepness are related, so that the radiative constraint tends to be tight when it does not let the tropopause move much and vice versa.

ZGV showed that these considerations were relevant for what is perhaps the simplest model that can determine its own tropopause: a dry, Boussinesq, primitive equation model on the beta plane forced by Newtonian relaxation to a Charney-like basic state (unbounded jet). Perhaps the main conclusion of that work was that the dynamics of tropopause height determination is profoundly affected by the properties of the radiative constraint, which in turn is a function of the heating formulation. In this paper we extend that previous work to the more complex and more realistic case of gray radiation forcing, with the underlying goal of understanding what determines the tropopause height. Apart from the different forcing used we keep nearly all the other simplifications made in $\mathrm{ZGV}$, including the neglect of a boundary layer scheme (more crucial for a model forced from the surface) and the use of a Boussinesq fluid.

The structure of this paper is as follows. Section 2 introduces our model and characterizes its radiative constraint. Section 3 describes in detail the climatology of the control run, focusing on its upper-tropospherelower-stratosphere (UTLS) region and on the structure of the tropopause. As we shall see, the gray radiation model can produce a more realistic tropopause than the Newtonian model: the thermal tropopause can be defined for typical parameters and displays a break at the latitude of the jet. Section 4 discusses the sensitivity of tropopause height in our model when parameters are varied and analyzes consistency with the radiative constraint and marginal criticality. Finally, section 5 concludes with a short summary and some discussion of these results.

\section{Model description}

We use a Boussinesq model in a channel, based on the Massachusetts Institute of Technology (MIT) GCM dynamical core (Marshall et al. 1997 and http://mitgcm. org/), in a hydrostatic, beta-plane setting, using a linear equation of state obtained linearizing the gas law (thermal expansion coefficient $\alpha_{T}=3.33 \times 10^{-3} \mathrm{~K}^{-1}$; see $\mathrm{ZGV}$ ). The dissipative terms include linear friction over a boundary layer with fixed depth, a sponge damping the eddies near the model top, and biharmonic diffusion. The geometry $\left(L_{X}=32000 \mathrm{~km}, L_{Y}=12500 \mathrm{~km}\right.$, $\left.H_{T}=40 \mathrm{~km}\right)$ and resolution $(128 \times 48 \times 80$, with a variable vertical grid) are also the same as in ZGV and diagnostics were obtained averaging the last 300 days from 600-day simulations.

The main difference from $\mathrm{ZGV}$ is the choice of the heating formulation, for which we use gray radiation with a standard two-stream scheme:

$$
\begin{aligned}
& \frac{d U}{d \tau}=U-B \text { and } \\
& \frac{d D}{d \tau}=B-D,
\end{aligned}
$$

where $U$ and $D$ are the upward and downward infrared radiative flux and the usual $3 / 2$ geometrical factor in this expression has been absorbed into the definition of $\tau$. The optical depth is defined as a function of height by the following equation: 


$$
\tau=\tau_{s}\left\{\frac{\alpha\left[\exp \left(-z / H_{a}\right)-\exp \left(-H_{T} / H_{a}\right)\right]+(1-\alpha)\left[\exp \left(-z / H_{T}\right)-\exp (-1)\right]}{\alpha\left[1-\exp \left(-H_{T} / H_{a}\right)\right]+(1-\alpha)[1-\exp (-1)]}\right\},
$$

where $\tau_{s}$ is the surface optical depth. Optical depth has two components: the first one decays with some "absorber" scale height $H_{a}$ and the second one with the depth $H_{T}$ of the computational domain. This latter component was introduced, following Frierson et al. (2006), to prevent exceedingly long radiative time scales in the upper atmosphere. The parameter $\alpha$ controls the relative importance of both components and is kept fixed in this study $(\alpha=0.9)$.

The boundary conditions that we use are also standard: $D(0)=0$ and $U\left(\tau_{s}\right)=\sigma T_{s}^{4}$, where $T_{s}$ is the surface temperature. However, we must use a modified version of the Stephan-Boltzmann law to calculate thermal emission. This is necessary because temperature always increases with height in our Boussinesq model when the fluid is stably stratified (temperature and potential temperature are equivalent in our formulation), which would translate to an increasing $B(z)$ when the standard law is used. Since increasing emission is only possible in the presence of internal heating, such a model would never be able to produce a radiative equilibrium stratosphere (convection would reach the top). Recognizing that our $T$ is more akin to potential temperature, we change its radiative role and introduce the following "cooling" temperature:

$$
T_{c}=T \times \exp \left(-\frac{\kappa z}{H_{\rho}}\right),
$$

where $H_{\rho}$ is a fictitious density scale height and $\kappa=2 / 7$. Temperature $T_{c}$ represents the temperature associated to potential temperature $\theta=T$ at height $z$ for a fluid with constant scale height $H_{\rho}$ (note that the real scale height is infinite in our incompressible model). We thus calculate thermal emission as

$$
B=\sigma T_{c}^{4}=\sigma T^{4} \times \exp \left(-\frac{z}{H_{c}}\right),
$$

where $\sigma$ is the Stefan-Boltzmann constant and $H_{c}=H_{\rho}$ ' $(4 \kappa)$ is an external parameter in the model. Note that by introducing a finite $H_{c}$, we essentially allow $T_{c}$ and $B$ to be decreasing functions of height, even when $T$ itself is not.

A final step involves the linearization of the emission law:

$$
\begin{aligned}
B & =\sigma T_{c}^{4}=\sigma T_{m}^{4}+4 \sigma T_{m}^{3}\left(T_{c}-T_{m}\right)+\cdots \\
& \approx \sigma T_{m}^{3}\left(4 T_{c}-3 T_{m}\right),
\end{aligned}
$$

where $T_{m}$ is a (height dependent) reference temperature. This is done to reduce ambiguity and facilitate the interpretation of the results when comparing simulations with very different mean temperatures (with the nonlinear law, temperature gradients decrease as the mean temperature increases, even when the differential heating does not change). We use for the reference temperature the radiative equilibrium temperature corresponding to some characteristic insolation $S_{0}=500 \mathrm{~W} \mathrm{~m}^{-2}$ :

$$
T_{m}=\left[\frac{S_{0}}{2 \sigma}(1+\tau)\right]^{1 / 4} .
$$

With this radiative model, the infrared radiative heating in the interior is given by

$$
Q_{R}=-\frac{d}{d z}(U-D) .
$$

The representation of the surface is an important aspect in any model forced from below. While it would be possible to develop an idealized boundary layer scheme along the lines of Frierson et al. (2006), we have rather aimed here to make our model as similar as possible to $\mathrm{ZGV}$ and to minimize the number of external parameters, even if that makes the destabilization in our model unrealistically strong. First, we neglect the mass of the surface, and assume that the shortwave heating received by the surface is immediately communicated to the atmosphere via convective and radiative fluxes. This leads to the following equation for the surface:

$$
S=F_{\mathrm{CONV}}+U_{s}-D_{s},
$$

where $S$ is the effective solar heating (after accounting for the albedo), $F_{\mathrm{CONV}}$ is the convective flux from the surface to the atmosphere, and $U_{s}, D_{s}$ are the upward and downward infrared fluxes at the surface. Additionally, we assume that the surface heat transfer coefficient $k_{s}$ is large enough that we can take $T_{s} \approx T(0)$ and calculate the convective flux $F_{\mathrm{CONV}}$ in the above equation using the lowest-model-level temperature to estimate $B_{s}$. Finally, we deposit all this flux at the lowest model level: $Q_{\mathrm{CONV}}=F_{\mathrm{CONV}} / \Delta z$, where $\Delta z$ is the vertical grid size of the lowest atmospheric level, and we prescind from a boundary layer scheme (other than convective adjustment). The solar heating is defined through the following analytical expression:

$$
S=S_{m}-\Delta S \tanh \left(\frac{y-L_{Y} / 2}{\sigma_{Y}}\right),
$$


where $S_{m}$ is the mean solar heating, which varies by a factor $\pm \Delta S\left(\Delta S \leq S_{m}\right)$ over meridional distances of order $\sigma_{Y}$.

\section{Radiative constraint for this model}

The radiative constraint $H=f\left(\tilde{\theta}_{z}\right)$ provides a relation between the tropopause height and adjusted tropospheric stratification (assumed uniform), such that the outgoing longwave radiation (OLR) $I=U(0)$ is consistent with the horizontal transport problem. This is solved numerically as follows. For each stratification $\tilde{\theta}_{z}$ we consider a range of possible values of tropopause height, and for each of those heights we calculate the values of $U_{s}$ and $B_{s}$ at the surface integrating downward from the top. The tropopause height is determined by minimization of $\left|U_{s}-B_{s}\right|$, so that the lower-boundary condition is satisfied.

Assuming that the domain depth $H_{T}$ is much larger than all other heights (and taking $\alpha=1$ ), the heating formulation is defined by three different parameters: the absorber scale height $H_{a}$, the fictitious scale height $H_{c}$, and the optical depth at the surface $\tau_{s}$. Dimensional analysis then shows that the radiative constraint may be expressed in the form

$$
\frac{H}{H_{c}}=f\left[\tau_{s}, \frac{H_{a}}{H_{c}}, \tilde{\theta}_{z} H_{c}\left(\frac{\sigma}{I}\right)^{1 / 4}\right] .
$$

Because of the large number of parameters appearing in the gray radiation formulation, the sensitivity of the radiative constraint on external parameters is more complex than for the Newtonian cooling model. Figure 1 describes the topology of this constraint obtained from numerical calculations as described above. Figures 1a and $1 \mathrm{~b}$ display the radiative constraint $H=f\left(\tilde{\theta}_{z}\right)$ in dimensionless form as a function of the two dimensionless parameters $H_{a} / H_{c}$ and $\tau_{s}$. As with most heating models, the tropopause height rises with increasing stratification. The radiative constraint is relatively insensitive to $\tau_{s}$ except in the small stratification limit, when the tropopause height rises with increasing $\tau_{s}$. On the other hand, the sensitivity on $H_{a}$ is nonmonotonic, with the tropopause dropping (rising) with increasing values of this parameter when $H_{a} / H_{c}$ is larger (smaller) than about 0.5 . The tropopause height always increases with $H_{c}$.

An important property of the radiative constraint is its steepness:

$$
s_{H}=\frac{\partial \log H}{\partial \log \tilde{\theta}_{z}}
$$

which measures the local sensitivity of tropopause height on stratification (see $\mathrm{ZGV}$ ). Small values of $s_{H}$ are associated with flat tropopause heights, while large values imply that the tropopause height is very sensitive to changes in stratification. Figures $1 \mathrm{c}$ and $1 \mathrm{~d}$ display the sensitivity of steepness on the external parameters. We can see that steepness increases with stratification and with $H_{a} / H_{c}$, while it is fairly insensitive to $\tau_{s}$. Over the range of parameters considered, the largest values of $s_{H}\left(s_{H} \approx 2\right)$ are obtained for $H_{a} / H_{c} \approx 1.4$ and $\tilde{\theta}_{z} H_{c}(\sigma / I)^{1 / 4} \approx 0.17$. However, note that this value of $s_{H}$ is much smaller than obtained in ZGV for the Newtonian cooling model, for which $s_{H} \rightarrow \infty$ in the loose limit. There is not such a thing as a "free floating" tropopause limit in this model.

Finally, Figs. 1e and 1f show the tightness of the radiative constraint, defined as

$$
\mathcal{T}=1-\frac{\tilde{\theta}_{z}}{\theta_{z_{R}}(H)},
$$

where $\theta_{z_{R}}(H)$ is the radiative equilibrium stratification at the tropopause. As discussed in $\mathrm{ZGV}$, this parameter measures how important is the vertical heat transport for the mean state. When tightness is small, the mean stratification does not differ much from radiative equilibrium, implying that the dynamical adjustment is weak. In that limit, we expect that the radiative constraint will not constrain much the flow and we refer to this as a loose radiative constraint. The opposite is true when $\mathcal{T}$ approaches 1 . Figure 1 shows that there is a strong relation between $\mathcal{T}$ and $s_{H}$, so that the radiative constraint is loose when the sensitivity of tropopause height on stratification is steep and tight when the sensitivity is weak. The same was found for the Newtonian cooling model in $\mathrm{ZGV}$.

\section{Description of the control run}

We choose the following combination of parameters, broadly representative of the extratropical troposphere, for our control simulation: $S_{m}=500 \mathrm{~W} \mathrm{~m}^{-2}, \Delta S=$ $300 \mathrm{~W} \mathrm{~m}^{-2}, \tau_{s}=4, H_{a}=2 \mathrm{~km}, H_{c}=7 \mathrm{~km}, f_{0}=10^{-4} \mathrm{~s}^{-1}$, $\beta=1.6 \times 10^{-11} \mathrm{~m}^{-1} \mathrm{~s}^{-1}$, and $\sigma_{Y}=1000 \mathrm{~km}$. To ensure that our results are not sensitive to resolution, this control simulation was repeated doubling the number of $X$ and $Y$ grid points and increasing the number of vertical grid points by $50 \%$, obtaining very similar results to those shown below. Likewise, the climatology obtained averaging the last 300 days from a 600 -day simulation changes very little when the simulation is extended for an additional 2400 days. Thus, all other simulations are only run for 600 days and only at the standard resolution.

Figure 2 shows some diagnostics for the control simulation. Although the circulation has a realistic structure, it 

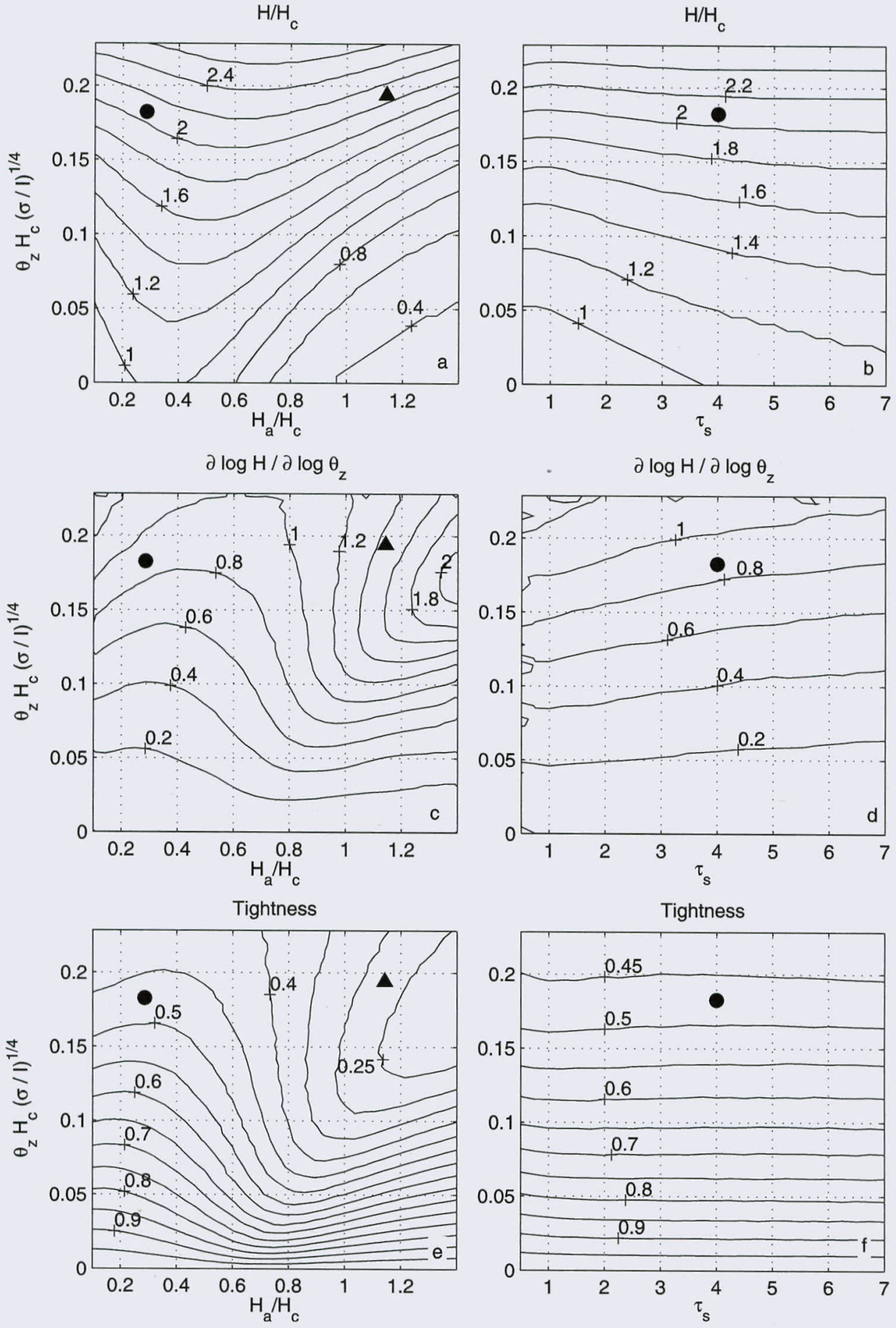

FIG. 1. Radiative constraint for the gray radiation model. Tropopause height as a function of stratification when (a) $H_{a} / H_{c}$ is varied and (b) $\tau_{s}$ is varied. (c),(d) As in (a),(b), but for the steepness of tropopause height. (d),(e) As in (a),(b), but for the tightness of the radiative constraint. The circle and triangle markers are the control simulation and a simulation with $H_{a}=8 \mathrm{~km}$, respectively. 

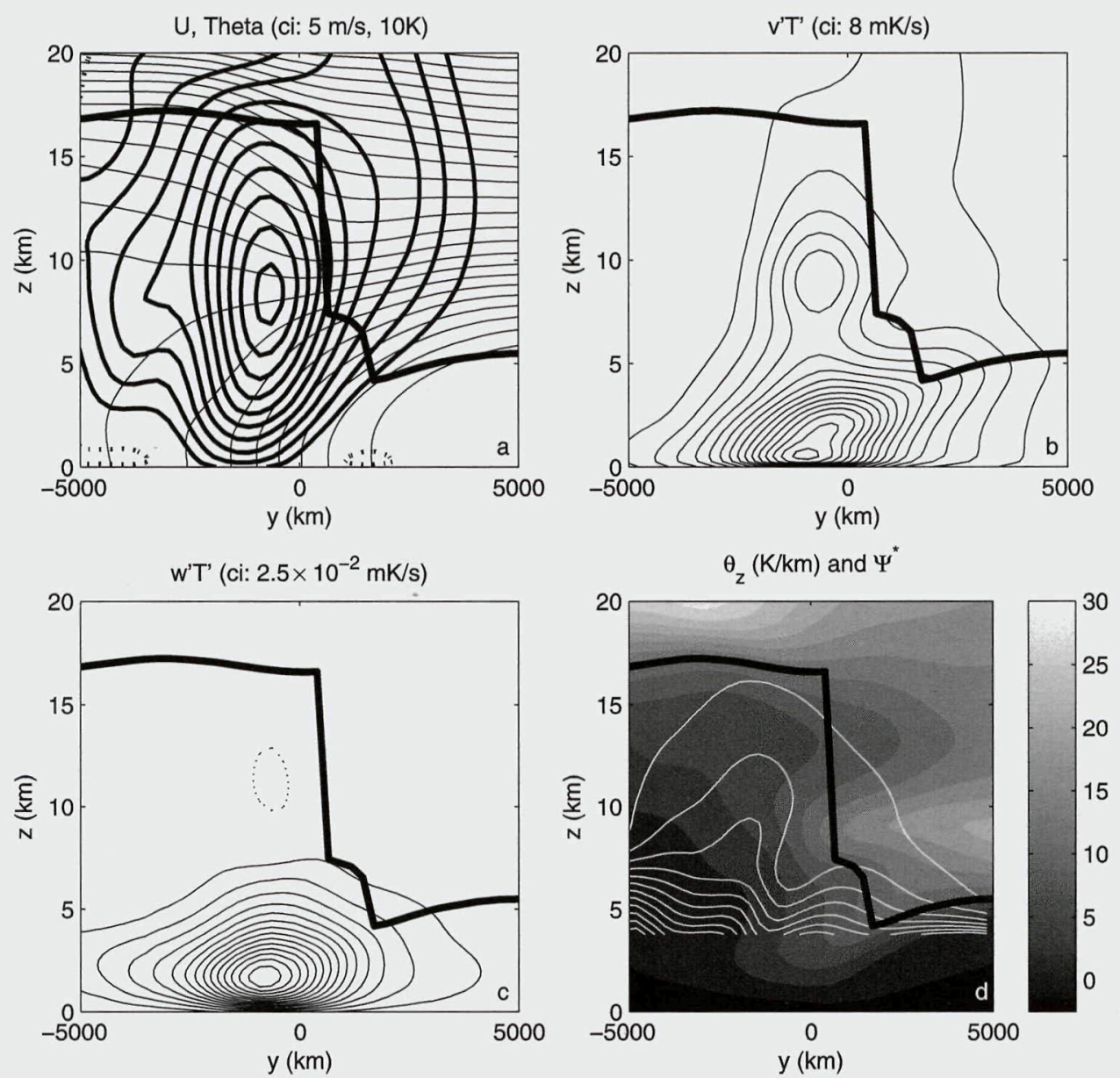

FIG. 2. Description of the equilibrium state for the control run. (a) Mean zonal wind (thick) and potential temperature (thin); (b) meridional eddy heat flux; (c) vertical eddy heat flux; (d) stratification (shading) and residual circulation (contours). Contour intervals are indicated in panel titles and the thermal tropopause is shown with thick line in all panels.

is also much stronger than observed and than the dry limit of Frierson et al. (2006), with similar parameters. The latter is likely due to the small thermal inertia in our model, which uses a massless surface, though other differences in the formulation, like the weak momentum mixing in the absence of a boundary layer scheme, might also play a role. On the other hand, the flow is more realistic than for the Newtonian cooling simulations presented in ZGV. While the zonal flow in that study was characteristic of the Charney problem (an unbounded jet), the baroclinicity now has a finite vertical scale and the jet stream displays an upper-tropospheric maximum (Fig. 2a). Above this level, the heat transport by the residual circulation exceeds the diabatic forcing of baroclinicity and the meridional temperature gradient reverses.

Other realistic features absent in the Newtonian model include a break in the thermal tropopause over the jet stream region ${ }^{1}$ and a secondary eddy heat flux maximum in the upper troposphere. The tropopause depicted in Fig. 2 was estimated applying the World Meteorological Organization (WMO) criterion (World Meteorological Organization 1957) to the cooling temperature $T_{c}$ as the lowest level at which $-\partial T_{c} / \partial z$ drops to and stays below $2 \mathrm{~K} \mathrm{~km}^{-1}$ over a $2-\mathrm{km}$ layer. There is of course nothing fundamental about the WMO definition and it cannot be expected to be meaningful for all

\footnotetext{
${ }^{1}$ An important qualitative difference with observations is that the high tropopause is observed over the region of baroclinic eddy generation (the region with surface westerlies and large eddy heat flux) in our model, rather than being confined to the tropics as observed. A possible explanation for this difference is that the jet maximum is extratropical in our model, while in observations the tropical forcing might displace the jet and tropopause break equatorward.
} 
climates and models. However, it is apparent in Fig. 2a that the WMO definition provides a reasonably good estimate for the thermal tropopause, as the stratification transitions from typical upper-tropospheric to lowerstratospheric values around that height. We will show below that the WMO criterion (when applied to $T_{c}$ ) can actually capture quite well the variability of tropopause height in our model when parameters are varied, for reasons explained later. Another important difference with the Newtonian cooling model is the larger $\overline{w^{\prime} \theta^{\prime}}$ to $\overline{v^{\prime} \theta^{\prime}}$ ratio observed here, consistent with the steeper isentropic slopes found over the lower troposphere.

Figure 3 describes in some detail the vertical structure of UTLS region averaged over the "baroclinic zone," defined here as the latitudinal band where the vertically integrated meridional eddy heat flux is within a $75 \%$ of its maximum value (this is shown in Fig. 5 for the control run). The UTLS region is more visible in our model than in observations, presumably because of our use of a Boussinesq formulation. In particular, the wind maximum and the thermal tropopause are separated by an unrealistically wide region with roughly half the mass of the tropospheric region below. The eddy vertical heat flux changes sign above the level of maximum wind (Fig. 3b), consistent with the change in tilt of the isentropic slope (the mixing slope $\overline{w^{\prime} \theta^{\prime}} / \overline{v^{\prime} \theta^{\prime}}$ and the isentropic slope are aligned when the eddies are quasiadiabatic and linear; see, e.g., Held and Schneider 1999). This implies that positive eddy kinetic energy (EKE) generation only extends up to the wind maximum, while the UTLS region spanning from that level to the thermal tropopause is a region of EKE destruction. Consistent with this, the simulated EKE peaks near the wind maximum and decays aloft (Fig. 3c).

Attesting to the dynamical relevance of the wind maximum, there are other features in the mean flow and eddy fluxes roughly collocated with it. The isentropic potential vorticity (PV) gradient reaches a local maximum slightly below the wind maximum (Fig. 3d), consistent with the kinematic interpretation of the jet stream as a barrier to mixing, which gives rise to large $\mathrm{PV}$ gradients at the tropopause (Greenslade and Haynes 2008). We estimate the PV diffusivity (shown in Fig. 3e) dividing the meridional PV flux by the meridional PV gradient and define the mixing barrier as the region where this diffusivity is below some small, subjectively chosen threshold $\left(0.3 \times 10^{6} \mathrm{~m}^{2} \mathrm{~s}^{-1}\right)$ - this barrier is shown shaded in all panels of Fig. 3. Figure $3 \mathrm{f}$ shows that the PV fluxes vanish over that region and recover aloft, although they still remain much weaker than in the lower troposphere. The nonmonotonic behavior in the eddy PV flux results from its baroclinic component, as the vertical Eliassen-Palm flux $\overline{f v^{\prime} \theta^{\prime}} / \bar{\theta}_{z}$ flattens locally over the barrier (Fig. 3h) and its convergence weakens. The weakening of the baroclinic eddy PV flux can be traced back to the upper-troposphere eddy heat flux maximum but local changes in the stratification are important for broadening this region with reduced PV flux. A layer with enhanced stratification (Fig. $3 \mathrm{~h}$ ) collocated with the eddy heat flux maximum is produced by the dynamical warming by the residual circulation, which adopts a more vertical orientation above that level (Fig. 2d). The change of character in the residual circulation is also apparent in the isentropic mass flux (shown in Fig. $3 \mathrm{i}$ averaged over the baroclinic zone and interpolated to height coordinates) - the pronounced kink at that level separating regions with fast and slow meridional flow.

Although the vertical eddy heat flux (Fig. 3b) and meridional eddy PV flux (Fig. 3f) are much weaker above than below the wind maximum, these weak fluxes have a major impact on the mean state because optical depth is small and radiative times large over the UTLS region. The former mixes the tropospheric stratification over a deep region (Fig. $3 \mathrm{~h}$ ), raising the thermal tropopause (also included in all panels of Fig. 3) well above the wind maximum. The latter sharpens the PV gradient at the jet maximum. This can be seen in Fig. 4, which shows the full 2D structure of the PV flux over the UTLS region. Strikingly, the PV flux changes sign and becomes upgradient collocated with and poleward of the jet stream maximum (as has also been noted in the extratropical troposphere; see Birner and Thompson 2011), so that the near vanishing of the PV flux in Fig. 3f only really occurs in the meridional average. This meridional dipolar pattern is due to the eddy momentum flux component of the PV flux (not shown). The PV flux reaches its maximum, positive value near but slightly northward of the jet stream maximum; this pattern is such that the basic state PV is decreased (increased) southward (northward) of the maximum and its meridional gradient sharpens (Fig. 5).

Figure 5 compares various tropopause definitions for our control run. As noted earlier, the thermal tropopause is located much higher than the level of maximum wind, which tops the region of positive EKE generation. The isentropic circulation slows down above that level and displays a kink, also shown in the figure. Interpreting the troposphere as the region over which the isentropic circulation drives the mean state off radiative equilibrium, Schneider (2004) proposed a tropopause definition based on the depth at which this circulation drops to a certain fraction of its maximum value at each latitude. The height of the mass flux tropopause for our control run is sensitive to the specific threshold used but lies in general somewhere between the zonal wind 
U
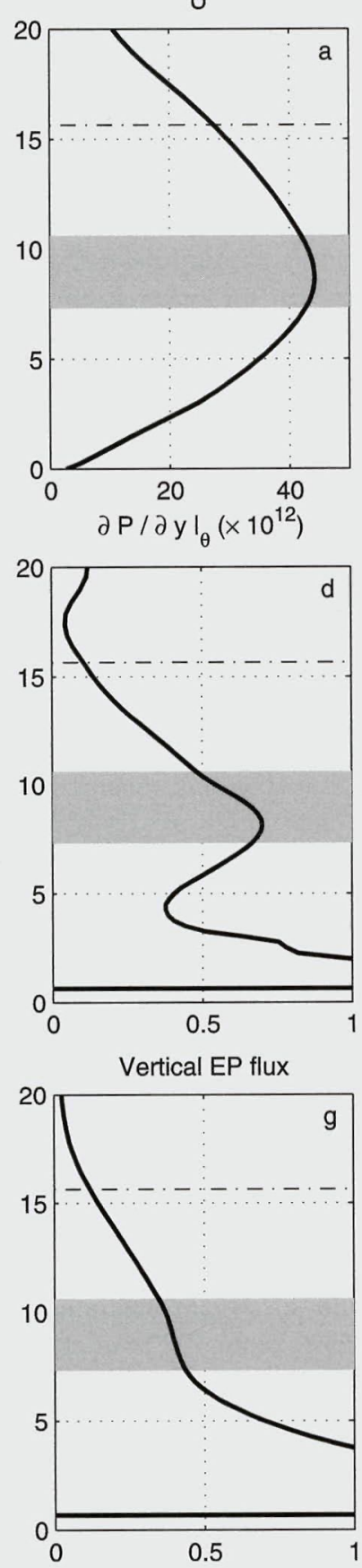
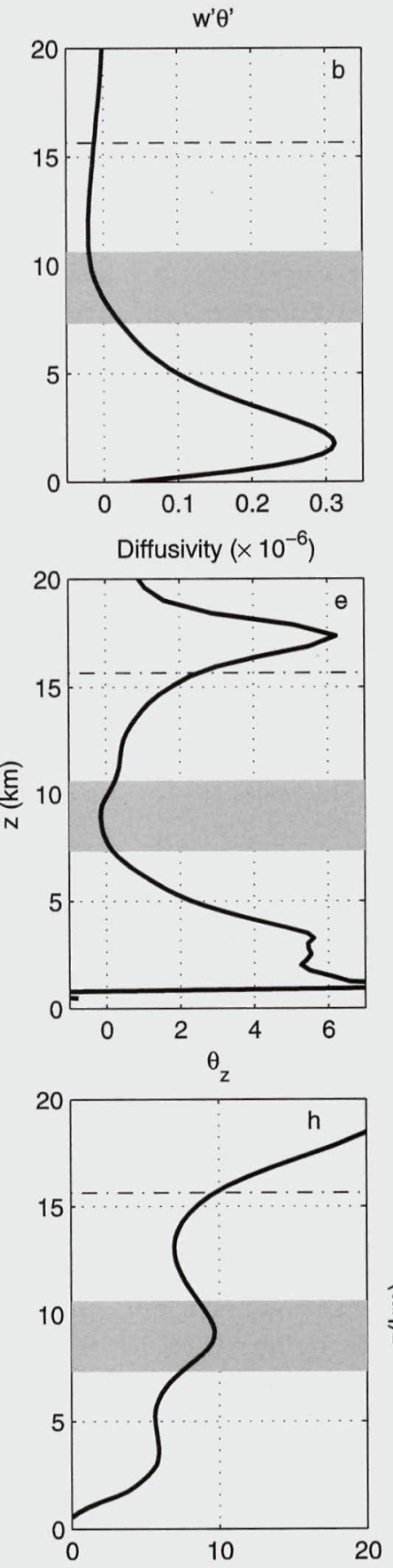
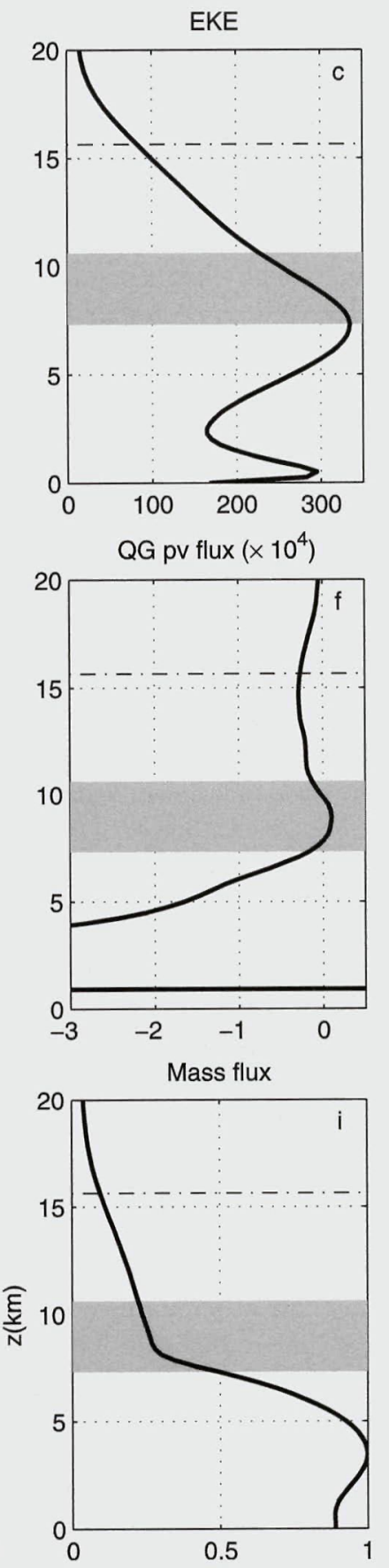

FIG. 3. For the control run, vertical profiles averaged over the baroclinic zone of (a) zonal wind, (b) vertical eddy heat flux, (c) eddy kinetic energy, (d) isentropic potential vorticity gradient, (e) empirical eddy diffusivity, (f) quasigeostrophic eddy PV flux, (g) vertical Eliassen-Palm flux, (h) stratification, and (i) isentropic mass flux, interpolated to height coordinates. The shaded band emphasizes the region with small diffusivity [see (e)] and the dashed-dotted line indicates the location of the thermal tropopause.

maximum and the thermal tropopause. Finally, the dynamical tropopause is often defined in terms of a constant PV surface in the real atmosphere. In our model, the thermal tropopause is well approximated by the 1-PVU surface. This value is smaller than typically used in the real atmosphere (and the troposphere-stratosphere PV transition not as sharp; not shown) because of the different structure of heating and density in our model.

Finally, we discuss the application of the radiative constraint for this simulation, which has tightness $\mathcal{T} \approx 0.48$ and 


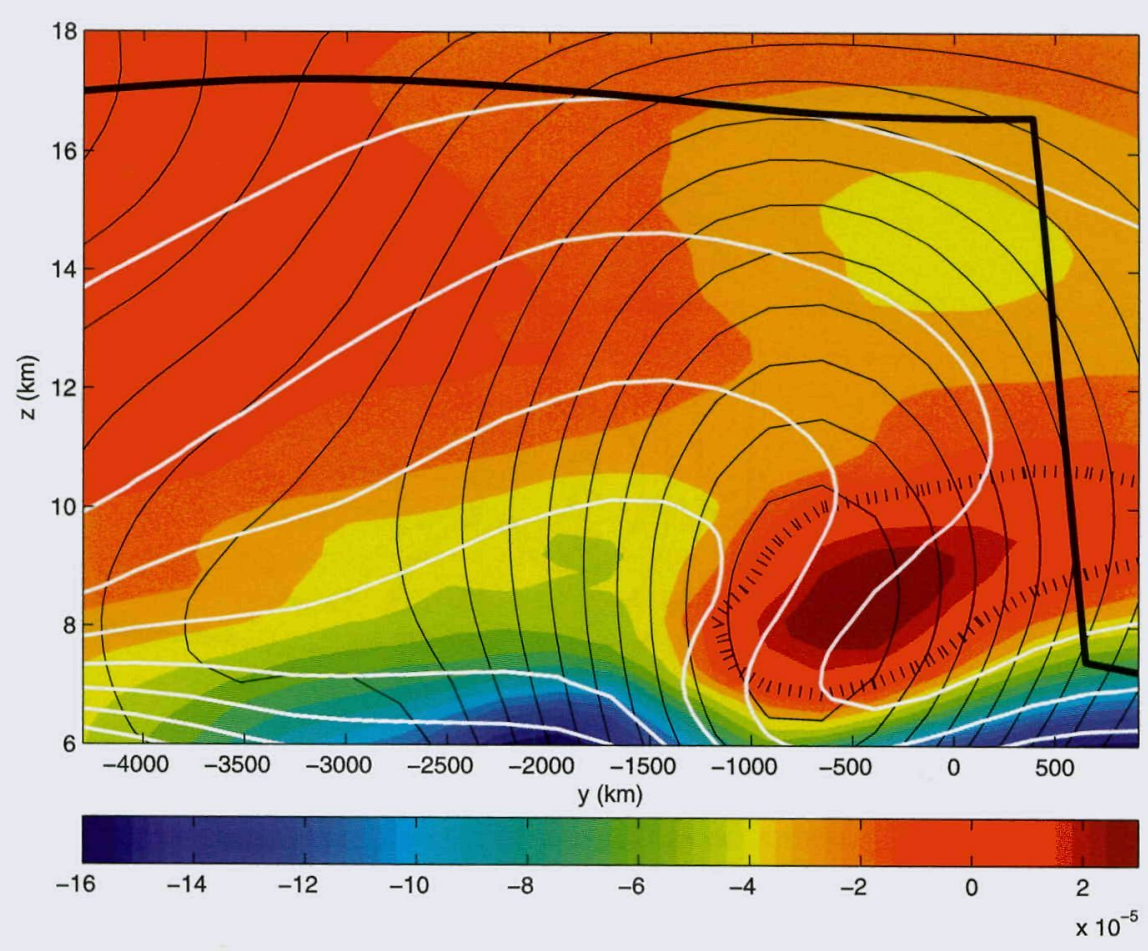

FIG. 4. The 2D structure over the UTLS region. The shading shows the quasigeostrophic PV flux $\left(\mathrm{s}^{-1}\right.$; with the zero contour thick vertical dash), the black contours the zonal-mean zonal wind, and the white contours the residual circulation. The black thick line is the thermal tropopause.

is mapped with a filled circle in Fig. 1. Figures 6a and 6b show the vertical thermal structure at the latitude of maximum meridional heat transport for this run in terms of the cooling temperature and potential temperature. Figure $6 \mathrm{~b}$ shows that the stratification is not really uniform: the fluid is weakly stratified at the surface and there is also a shallow, highly stratified layer near the level of maximum zonal wind, as noted above. However, the stratification is still sufficiently different from radiative equilibrium that a sharp transition is observed between the tropospheric region with dynamically adjusted stratification and the stratospheric region in near-radiative equilibrium aloft. Since the dynamical adjustment in stratification in the troposphere is important for this simulation, it is not surprising that the radiative constraint works well, giving a prediction in good agreement with the thermal tropopause. Figure $6 \mathrm{a}$ also illustrates why the WMO criterion provides a reasonable estimate of the thermal tropopause for this run and, for similar reasons, in the real atmosphere. Essentially, the small optical depth at tropopause levels implies that a stratosphere in radiative equilibrium will be nearly isothermal (in our model, in terms of the cooling temperature). A $2 \mathrm{~K} \mathrm{~km}^{-1}$ lapse rate threshold provides good contrast with this condition and is also significantly more stable than typical tropospheric lapse rates when the troposphere is heated from below. The relevance of the WMO criterion for this setting is demonstrated in Fig. 6c, which shows that the WMO tropopause and mass flux tropopause scale roughly linearly over a broad range of parameters. In contrast, when the radiative constraint is loose (Figs. 6d-f) a thermal tropopause can no longer be defined and the WMO criterion fails to capture the depth of the isentropic circulation, as discussed in detail in section $4 \mathrm{a}$.

\section{Sensitivity of tropopause height}

We next discuss the sensitivity of tropopause height in our model when external parameters are varied. We study the sensitivity to the forcing changing $S_{m}(=300$, $\left.\mathbf{5 0 0}, 750,1000,1250 \mathrm{~W} \mathrm{~m}^{-2}\right)$ and $\Delta S(=100,150,200,250$, 300, $\left.350,400,450 \mathrm{~W} \mathrm{~m}^{-2}\right)$, the sensitivity to rotation changing $f_{0}\left(=\mathbf{1}, 1.5,2,2.5,3,3.5,4,5 \times 10^{-4} \mathrm{~s}^{-1}\right)$ and $\beta\left(=0.8,1.2,1.6,2,2.4,2.8,3.2 \times 10^{-11} \mathrm{~m}^{-1} \mathrm{~s}^{-1}\right)$, and the sensitivity to the radiative parameters changing $H_{a}(=1$, 2, 3, 4, 5, 6, 7, 8 km), $\tau_{s}(=1,2,4,6,8,10)$, and $H_{c}(=6,7$, $8,9,10,11,12 \mathrm{~km})$. Only one parameter is changed at each time, keeping all others at their control values (in boldface). The simulations varying $S_{m}$ are performed with and without the convective adjustment scheme while the rest of the simulations are only performed with convective adjustment. 


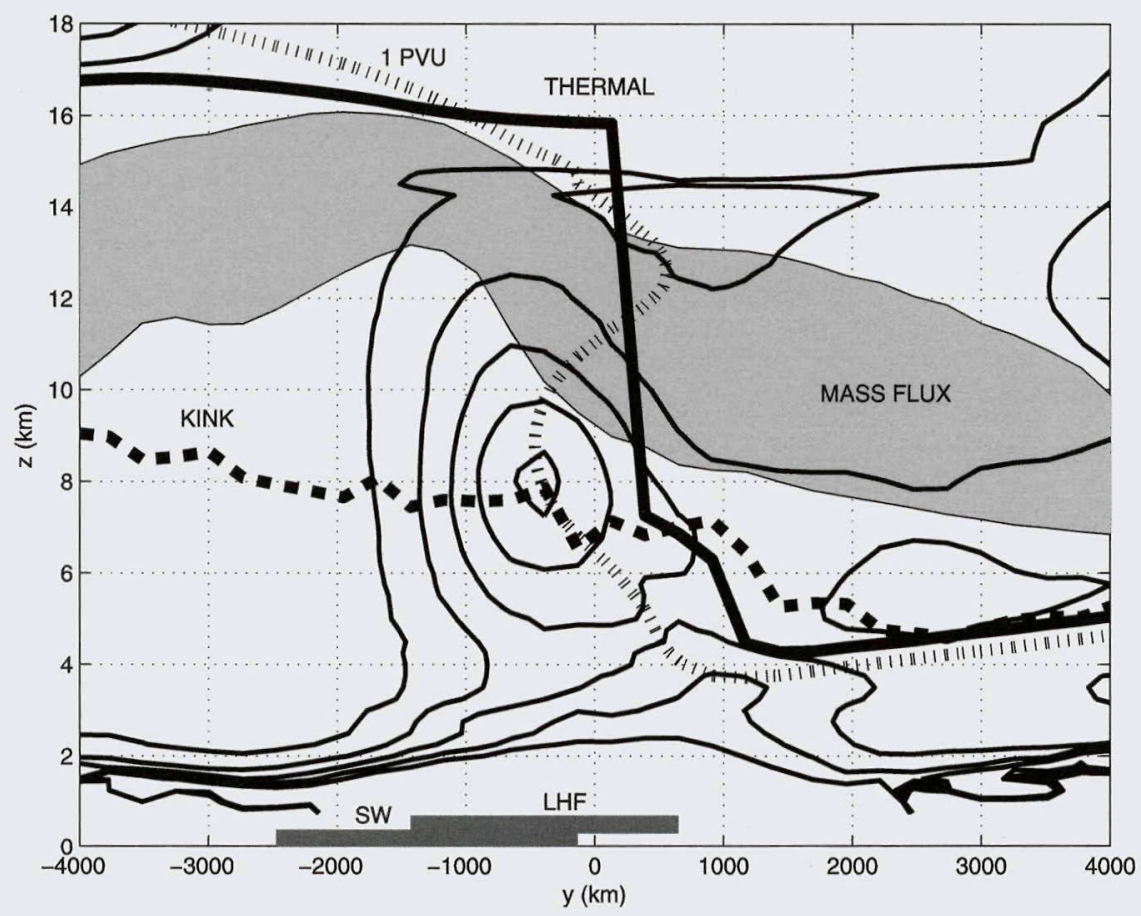

FIG. 5. Comparison of various tropopause definitions for the control run. The thick solid, dotted; and dashed lines show the thermal tropopause, the dynamical tropopause (1-PVU isoline), and the level at which the isentropic mass flux displays a kink (implying a slowdown of the circulation aloft). The shaded region defines the mass flux tropopause as the region where the isentropic mass flux drops to the range of $10 \%-20 \%$ of its maximum value at each latitude and the solid contours show isolines of the isentropic potential vorticity gradient, illustrating the formation of a mixing barrier. We also show for reference the location of the surface westerlies (SW) and the region with large eddy heat flux (LHF) used to define the baroclinic zone in our diagnostics.

The forcing is defined in our model by two parameters: the mean heating $S_{m}$ and the differential heating $\Delta S$. Zurita-Gotor and Vallis (2010) found that both parameters were important in a two-level study using a simplified version of the continuous gray radiation formulation described here. As $S_{m} / \Delta S$ was increased in that model the ratio between vertical and meridional eddy heat transport also increased, which translates into a steeper isentropic slope when the eddies are quasi adiabatic. However, we have found our model to be much less sensitive to $S_{m}$, as illustrated in Fig. 7. As $S_{m}$ increases there is some destabilization of the troposphere, but this is very weak-almost imperceptible in Fig. 7a. The dominant stratification signal is actually an increase over the UTLS region that makes the tropopause drop, which is consistent with the radiative constraint. The reason for the insensitivity of the tropospheric stratification is that when $S_{m}$ increases, the bulk of the increased vertical heat transport is accomplished by the infrared radiative flux (Fig. 7c), implying that the vertical destabilization and the eddy vertical heat flux vary little (Fig. 7b). The only exception is the surface boundary layer, a region over which the vertical transport by the convective adjustment scheme (Fig. 7d) is both important and dependent on $S_{m}$. The results are very similar when these simulations are repeated turning off the convective adjustment scheme (Figs. 7e,f), except that the vertical heat flux by the resolved convective motions now takes up the vertical heat transport carried by the convective adjustment scheme before. However, this only impacts the stratification over a very shallow surface layer. We thus conclude that the strong sensitivity to $S_{m}$ displayed by the simple model of Zurita-Gotor and Vallis (2010) is unrealistic because of its neglect of infrared radiative feedbacks and use of a fixed (deep) convective depth.

Figure 8 explores the sensitivity of the various tropopause definitions introduced in section 3 to changes in other external parameters in the model. We only represent the height of maximum zonal wind (dashed line with square markers) in that figure, but note that the level of maximum isentropic PV gradient and the kink in the isentropic mass flux scale well with this height, with the following two exceptions. For moderate to large $f_{0}$ there is no PV gradient maximum over the UTLS region 

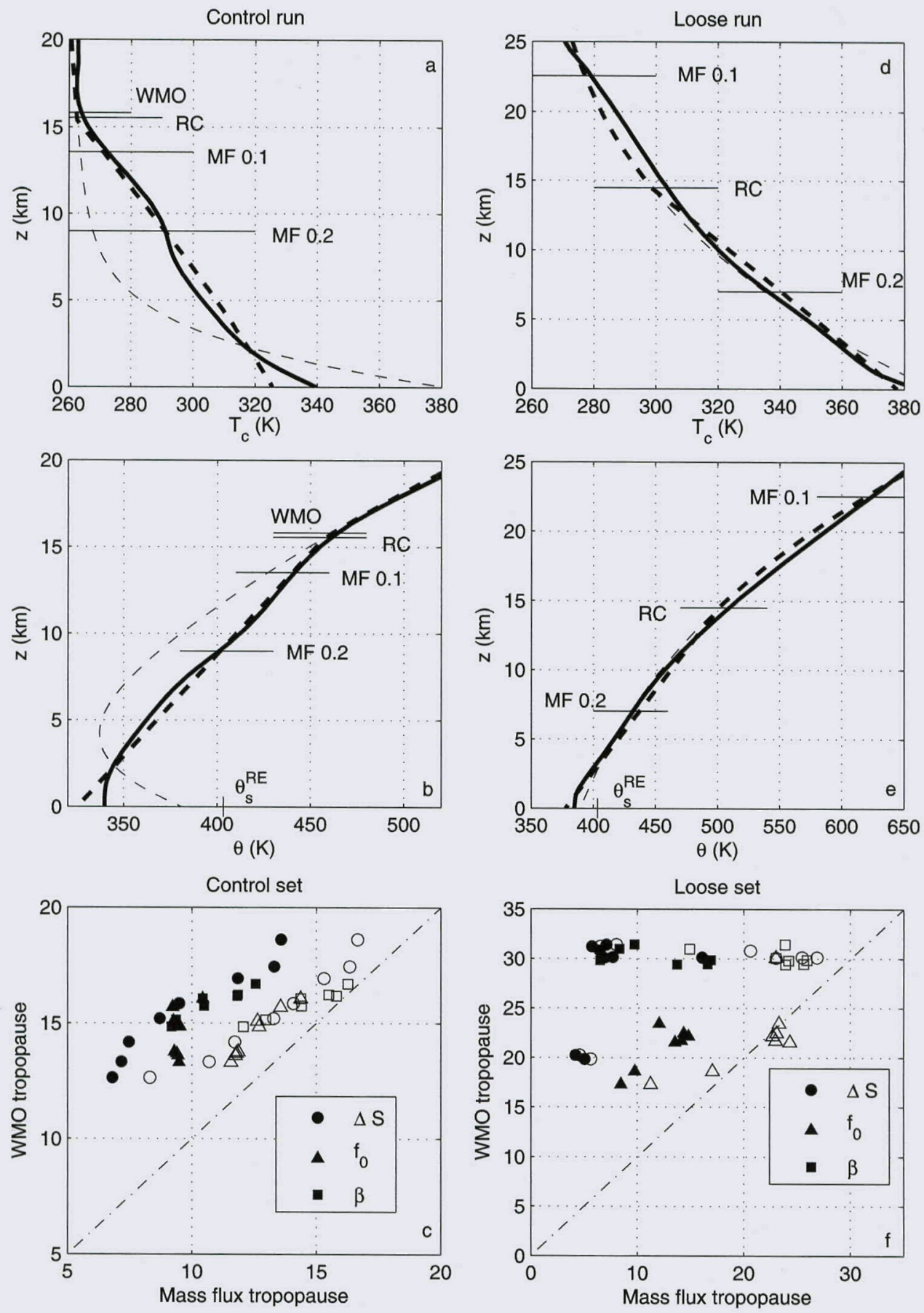

FIG. 6. (a) For the control run at the latitude of maximum meridional heat flux: cooling temperature $T_{c}$ simulated by the model (thick solid), in radiative equilibrium (thin dashed), and in the radiative constraint idealization (thick dashed). (b) As in (a), but for potential temperature; the surface radiative equilibrium temperature $\theta_{s}^{\mathrm{RE}}$ associated with the thin dashed profile is also shown. We indicate in both panels the WMO tropopause, the mass flux tropopause ( 0.1 or 0.2 mass flux isolines), and the tropopause height predicted by the radiative constraint (RC). (c) Scatterplot of the WMO tropopause against the mass flux tropopause defined using a 0.2 (filled marker) or 0.1 (empty marker) threshold, when the parameters indicated in the legend are varied. (d)-(f). As in (a)-(c), but for the loose simulation with $H_{a}=8 \mathrm{~km}$. 

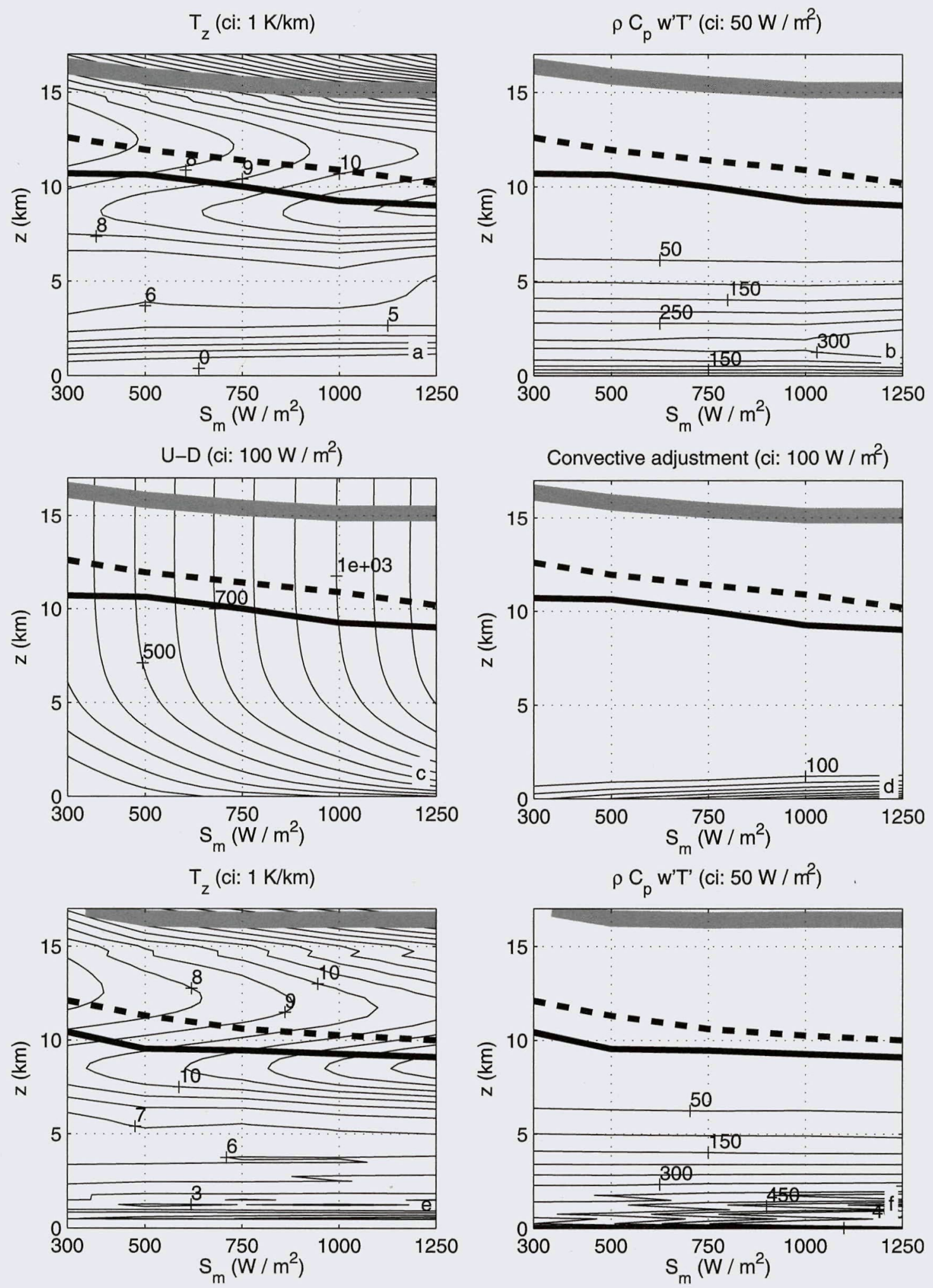

FIG. 7. For the simulations varying $S_{m}$ : (a) mean stratification; (b) vertical heat transport by the resolved eddy fluxes; (c) radiative infrared heat flux; (d) inferred heat transport by the convective adjustment scheme; and (e),(f) as in (a),(b), but in simulations run without convective adjustment. The thermal tropopause is shown with broad gray line, the mass flux tropopause ( 0.2 isoline) with thick solid line, and the radiative constraint prediction with thick dashed line. Contour intervals are displayed in panel titles and the $S_{m}$ variations simulated are indicated by the horizontal grid. 
and the isentropic mass flux no longer exhibits a kink. Additionally, while the level of maximum wind and PV gradient increases with $H_{a}$ (Fig. 8d) the height of the isentropic mass flux kink exhibits no variations (not shown). Figure 8 shows that the level of the wind maximum is largely controlled by the radiative scale heights $H_{c}$ and $H_{a}$, changing relatively little with other parameters. In contrast, the thermal tropopause (thick dark gray line) and the mass flux tropopause (light gray band, encompassing regions where the isentropic flux is within $10 \%-20 \%$ of its maximum) also increase with $H_{c}$ but are sensitive to other parameters as well. The thermal and mass flux tropopause definitions generally scale well with each other (see also Fig. 6c) though the thermal tropopause may not be well defined for all radiative parameters.

Figure 8 also compares the sensitivity of the thermal and mass flux tropopause estimates with predictions based on the radiative constraint $H=H_{\text {rad }}\left(\theta_{z}\right)$ (solid) and the dynamical constraint of marginal criticality (dashed). The latter essentially requires that the tropopause height scales as the Charney height (Held 1978; see ZGV for more details):

$$
H=h=-\frac{f_{0}}{\beta} \frac{\partial_{y} \theta}{\partial_{z} \theta} .
$$

All estimates and predictions represent meridional averages over the baroclinic zone defined in the previous section. Additionally, we use tropospheric averages (from the surface to the 0.2 mass flux isoline) to estimate characteristic meridional and vertical temperature gradients. Since estimates based on tropospheric averages incorporate information on the simulated tropopause height and may bias our assessment of the predictors, ${ }^{2}$ we also plot in Fig. 8 with thin solid/dashed lines predictions based on the local gradients at a fixed height of $5 \mathrm{~km}$ (for clarity, these fixed-height estimates are only included in those panels where they are deemed informative).

Figure 8a shows that the thermal and mass flux tropopause estimates increase uniformly with differential

\footnotetext{
${ }^{2}$ Because the stratification typically increases with height, a spurious increase in our estimate for tropopause height will tend to bias the tropospheric average stratification to larger values, which would produce some semblance of agreement with the radiative constraint even when the mean state does not change. The reverse is true for the marginal criticality prediction that the tropopause height scales with the isentropic slope: since this slope flattens with height, the mean tropospheric isentropic slope will decrease when using a larger tropopause height, producing a fictitious anticorrelation.
}

heating $\Delta S$. This tropospheric expansion is in good agreement with predictions by the radiative constraint (solid lines), as tropospheric stratification (both vertically integrated and at a fixed level) increases with differential heating. In contrast, the marginal criticality predictor clearly fails to reproduce the simulated sensitivity of tropopause height, as the tropospheric average isentropic slope decreases with increasing differential heating. This is partly due to the bias in the estimate noted earlier, as the isentropic slope at fixed height changes little with $\Delta S$ between the top of the boundary layer and the wind maximum. However, even with this convention marginal criticality fails to explain the simulated increase in tropopause height. On the other hand, the tropopause height is only weakly sensitive to $f_{0}$ and $\beta$ (Figs. $8 \mathrm{~b}, \mathrm{c}$ ), which is in good agreement with the radiative constraint prediction because the stratification changes little in these runs. In contrast, the marginal criticality condition would predict large variations in the tropopause when $f_{0}$ and $\beta$ are independently varied, which is not observed in our simulations.

Finally, we discuss the sensitivity of tropopause height to changes in the radiative parameters $H_{a}, H_{c}$, and $\tau_{s}$. Note that though the WMO tropopause was found to be relevant for all previous simulations using realistic radiative parameters, the same may not be true when the radiative parameters are varied. This is, for instance, the case with large values of $H_{a}$ (Fig. 8d): while a tropopause can still be defined in that limit using the WMO criterion, this tropopause is very high up in the atmosphere and apparently unrelated to the depth of the circulation. Likewise, Fig. 8f suggests some divergence between the mass flux and thermal tropopause for large $\tau_{s}$. The sensitivity of tropopause height is very simple when $H_{c}$ (Fig. 8e) or $\tau_{s}$ (Fig. 8f) are varied. All tropopause estimates, including the wind maximum, increase linearly with $H_{c}$ (which may be interpreted as a vertical height rescaling), while the sensitivity on $\tau_{s}$ is weak because of our linearization of the emission law. The radiative constraint works well in both cases but marginal criticality only really works when $H_{c}$ is varied. In contrast, the sensitivity to $H_{a}$ is much more complex. The simulated mass flux tropopause exhibits nonmonotonic variations, rising (dropping) with $H_{a}$ for moderate (large) values of this parameter. This is consistent with the sensitivity displayed by the radiative constraint (Fig. 1a) and, indeed, the prediction based on that constraint is in qualitative agreement with the simulated variations, while the marginal criticality prediction is essentially anticorrelated. However, note that the opposite is true when fixed-height gradients are used to compute the estimates (thin lines), which suggests that changes in the vertical structure are important when 

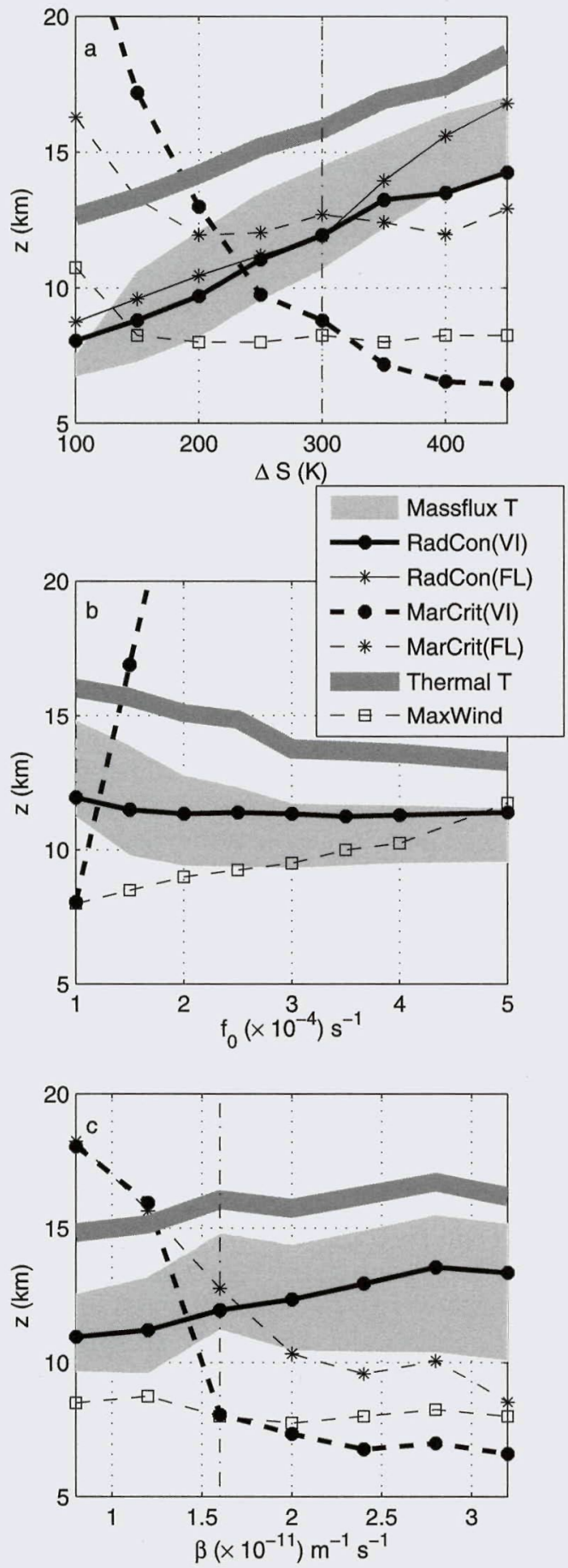
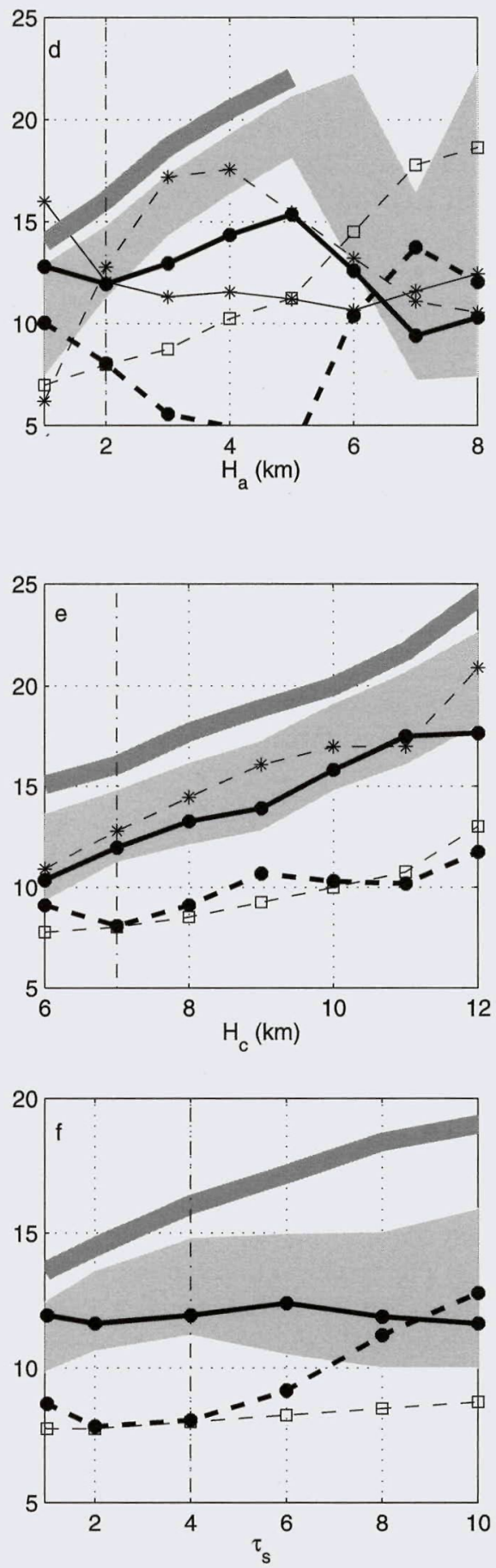

FIG. 8. Sensitivity of tropopause height and radiative and dynamical predictors when (a) $\Delta S$, (b) $f_{0}$, (c) $\beta$, (d) $H_{a}$, (e) $H_{c}$, or (f) $\tau_{s}$ is varied. The thick dark gray line indicates the thermal tropopause, the light gray band the mass flux tropopause (region where the isentropic mass flux is between $10 \%$ and $20 \%$ of its maximum), and the dashed line with square markers the height of the wind maximum. The solid line gives the radiative constraint prediction using either an averaged tropospheric stratification (thick solid) or the local stratification at a fixed level (thin solid; not shown in all panels). The thick and thin dashed lines show the same for the marginal criticality prediction. The control value of each parameter is indicated with a vertical axis line. 

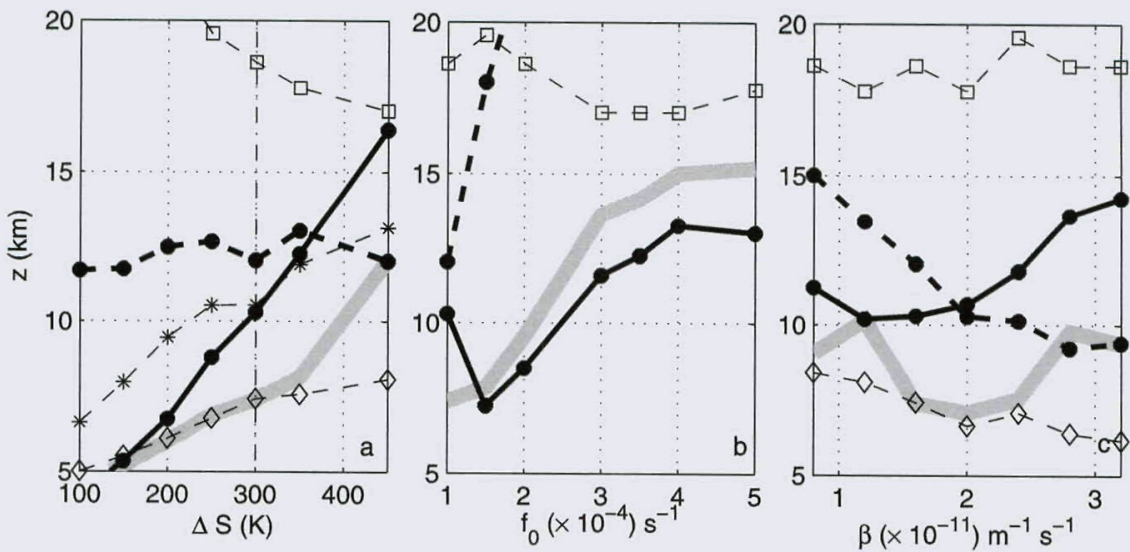

FIG. 9. Sensitivity of tropopause height and radiative and dynamical predictors with $H_{a}=$ $8 \mathrm{~km}$ when (a) $\Delta S$, (b) $f_{0}$, and (c) $\beta$ are varied. The thick light gray line indicates the mass flux tropopause ( 0.2 contour), the solid black line the radiative constraint prediction, and the dashed line the marginal criticality prediction (thick or thin as in Fig. 8). We also show the height of maximum wind (dashed line with black circles) and the level of the kink in the isentropic mass flux (dashed line with diamonds).

$H_{a}$ is varied. Another indication of these complex changes in vertical structure is that the height of the mass flux kink and the level of maximum wind/PV gradient no longer scale together: only the latter increases with $H_{a}$, while the former remains roughly constant (not shown).

\section{A loose limit}

The dependency of tropopause height and the predictors on changes in the forcing and rotation is very similar to that reported in ZGV using Newtonian heating for the strongly destabilized set $\left(\theta_{z_{0}}=-20 \mathrm{~K} \mathrm{~km}^{-1}\right)$. In contrast, the set with $\theta_{z_{0}}=0 \mathrm{~K} \mathrm{~km}^{-1}$ in that paper showed some tendency to adjust to marginal criticality. It was argued in ZGV that the different behavior between both sets was due to the fact that the radiative constraint only prevents the adjustment to marginal criticality for large vertical heat transport, when this constraint is tight. Specifically, the control run in ZGV had $\mathcal{T}=0.27$, compared to $\mathcal{T}=0.71$ for the $\theta_{z_{0}}=-20 \mathrm{~K} \mathrm{~km}^{-1}$ simulation. Our control simulation has tightness $\mathcal{T}=0.48$.

To test the robustness of the concepts introduced in $\mathrm{ZGV}$, we have used guidance from Fig. 1 to choose radiative parameters conducive to a loose radiative constraint. This can be achieved by considering unrealistically large absorber scale heights: Figs. $6 \mathrm{~d}$ and $6 \mathrm{e}$ show the vertical structure of the cooling temperature and potential temperature for a simulation with $H_{a}=8 \mathrm{~km}$ (mapped with a filled triangle in Fig. 1), which has tightness $\mathcal{T} \approx$ 0.28 . Consistent with this low tightness, the radiative equilibrium and adjusted profiles are almost indistinguishable now, making it difficult to identify the tropopause visually. Physically, this occurs because with this large value of $H_{a}$ the atmosphere is no longer optically thin at tropopause levels and the lower stratosphere is no longer isothermal. Thus, the transition to radiative equilibrium occurs at lapse rates of about $4.5 \mathrm{~K} \mathrm{~km}^{-1}$, poorly differentiated from typical tropospheric lapse rates in that region. The WMO lapse rate threshold of $2 \mathrm{~K} \mathrm{~km}^{-1}$, though reached higher up in the stratosphere, is no longer relevant for tropopause height in this setting, as illustrated by the lack of correlation between the mass flux and thermal tropopause when nonradiative parameters are varied in Fig. $6 f$.

Figure 9 describes the sensitivity of tropopause height to changes in the forcing and in rotation for this setting. In these simulations the isentropic mass flux has a very long tail, with the 0.1 isentropic mass flux contour separating widely from the 0.2 contour (cf. Fig. 8d) and becoming very noisy. Since the thermal tropopause is not well defined now, we use the 0.2 mass flux contour as our best reference for the simulated tropopause height. On the other hand, the wind and PV gradient maxima also move high up and are differentiated from the level of the mass flux kink (both are now shown in Fig. 9). Given the complexity of the vertical structure and the lack of definition of the tropopause, the comparison with the radiative and marginal criticality constraints must be made with caution. With these caveats, Fig. 9 suggests that while the radiative constraint still works qualitatively, marginal criticality works a bit better now than with the control setting. Our results are summarized in Fig. 10, which compares the predictive skill of the radiative and marginal criticality constraints for both sets using scatterplots. The mass flux tropopause and 

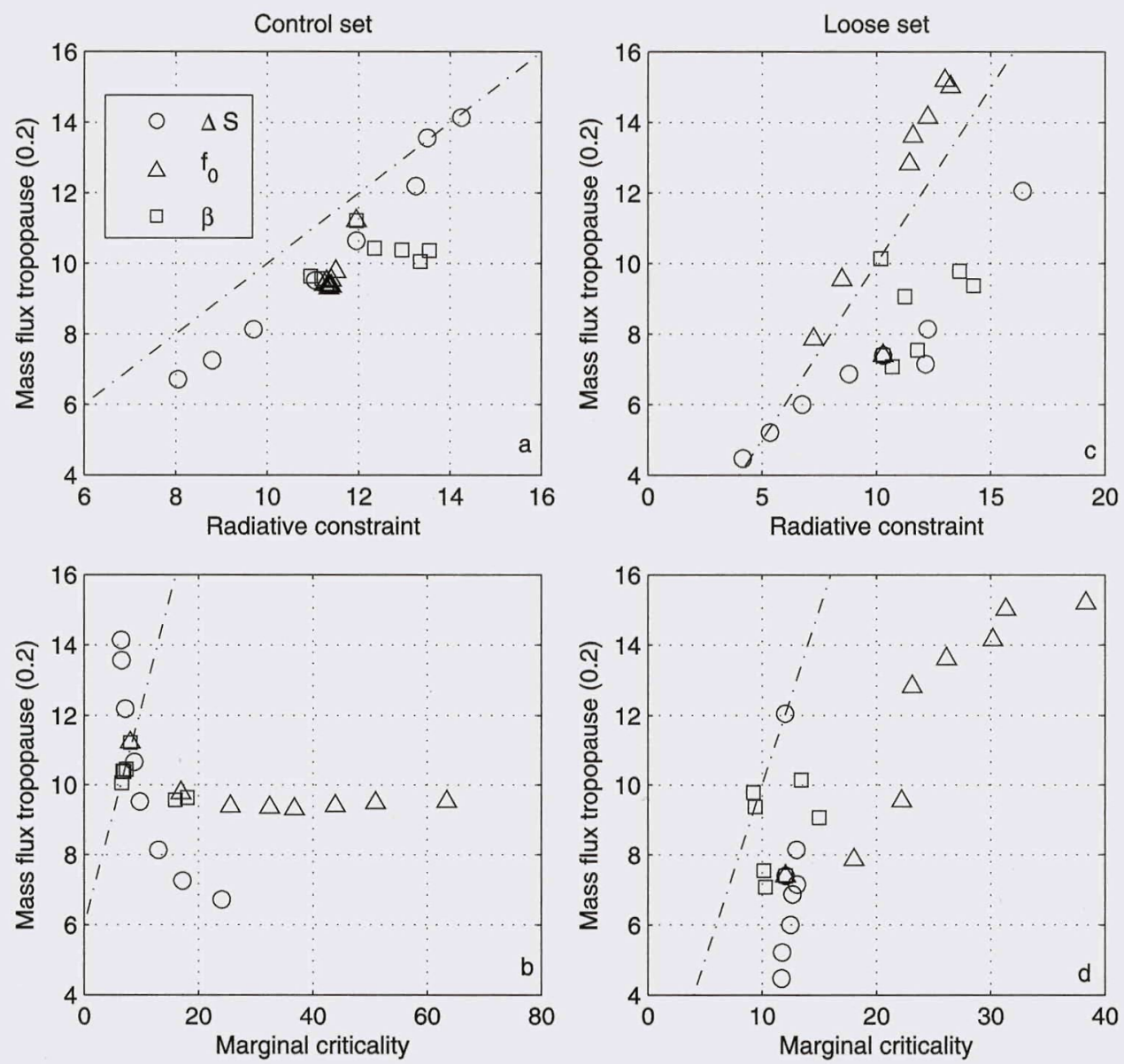

FIG. 10. (a) Scatterplot of simulated mass flux tropopause ( 0.2 contour interval) against the radiative constraint prediction for the control set, when the parameters indicated in the legend are varied; (b) as in (a), but for the marginal criticality prediction; and (c),(d) as in (a),(b), but for the loose setting with $H_{a}=8 \mathrm{~km}$.

radiative constraint estimate are positively correlated in both settings, though the scatter is larger when the radiative constraint is loose. On the other hand, the marginal criticality estimate is negatively correlated with variations in tropopause height in the control setting and positively correlated in the loose setting. However, note that even in that case, marginal criticality tends to overestimate the simulated variations in tropopause height.

\section{Discussion and conclusions}

The height of the tropopause is, in general, determined by a combination of diabatic forcing and essentially adiabatic dynamical effects related to the ability of the fluid to transfer heat sideways and upward by a combination of baroclinic eddies and convection. One would like to understand and even predict whether diabatic or dynamical (to use a convenient shorthand) effects are more important in practice, and to this end ZGV proposed a theoretical framework for understanding the relative roles played by the two effects. In this framework, the effectiveness of diabatic forcing manifests itself through the flexibility of the radiative constraint - the constraint that must be satisfied to satisfy the radiation budget at the top of the atmosphere. If the radiative constraint is "tight," meaning that small changes in the tropopause height would lead to large changes in outgoing radiation, then the tropopause height is effectively constrained by the thermal forcing. On the other hand, if the constraint is loose then dynamical effects are allowed a larger role. ZGV explored these ideas using an idealized model with Newtonian cooling. In this study we further extend and test the ideas using a model with a gray radiation scheme instead of Newtonian cooling, with the goal of understanding whether the radiative constraint is an effective one in a more realistic setting.

There are, of course, many possible definitions of the tropopause, differing both in detail and in concept. From the point of view of the radiative constraint, the most 
appropriate definition, and perhaps the most widespread in meteorology, is the thermal definition: the level at which the stratification transitions from typical tropospheric to stratospheric values. The WMO criterion (World Meteorological Organization 1957) is a particular case of this definition, appropriate for current atmospheric parameters and when the optical depth is small at tropopause levels and the lower stratosphere is near isothermal. However, it is not always possible to define a thermal tropopause because in some heating regimes, particularly when the radiative constraint is loose, there is no marked stratification contrast separating a "troposphere" from a "stratosphere," yet the lower atmosphere-the troposphere-is distinct from the stratosphere above by the presence of nonnegligible horizontal mass fluxes. This suggests that a mass flux definition (the depth of the isentropic circulation) is a more robust measure and we use that definition to probe the sensitivity of tropopause height on external parameters. This tropopause definition scales well with the thermal tropopause for those regimes in which the thermal definition is applicable.

Using the mass flux definition, our main results may be summarized as follows:

- The predictions of the idealized radiative constraint are, in general, in good agreement with the simulated sensitivity of tropopause height when external parameters are varied.

- Depending on the radiative parameters, the constraint may be tight and involve a significant adjustment of the tropospheric stratification and, consequently, a well-defined thermal tropopause, or it may be loose and produce a poorly defined one. When the constraint is tight it provides the most useful and quantitatively accurate predictions of tropopause height.

- The flow does not appear to adjust to marginal criticality except in the loose limit. In this limit, our results are less clear than with the Newtonian model because of the ambiguity in defining the tropopause with a more complex vertical structure. ${ }^{3}$

\footnotetext{
${ }^{3}$ Despite their comparable values of tightness, the vertical heat transport is much larger for our loose run than for the loose limit of the Newtonian cooling model, with the vertical heat transport only weakening by a factor of 1.5 when going from the tight to the loose limit, compared to a factor of 7.5 in the Newtonian cooling model. As a result, the adjustment to marginal criticality might not be as prevalent for the loose limit of this model as for the Newtonian heating model. Because radiative time scales are very long in the upper troposphere in the gray radiation model, tightness in this model is not as strongly tied to the strength of the lowertroposphere vertical heat flux (always large) as in the Newtonian cooling model.
}

- The gray radiation formulation produces a tight radiative constraint for typical radiative parameters and hence the model does not typically adjust to marginal criticality.

Overall, the results presented here and those of ZGV suggest that the surface destabilization/strength of required vertical transport is a key aspect of the heating formulation insofar as tropopause height and supercriticality are concerned. The sensitivity of these parameters in the Newtonian forcing model of $\mathrm{ZGV}$ is similar to that in the gray radiation model only when the Newtonian model is relaxed to a very unstable radiative equilibrium profile, but not when a convectively neutral profile is used. The contrast between these two cases may seem counterintuitive because when convection is much faster than baroclinic transport, one might expect that relaxation to a convectively adjusted state would produce similar results to letting convection act in a strongly destabilized setting. However, note that relaxing to a neutral state is equivalent to assuming that the implied convective transport-the difference between relaxing to an unstable and an adjusted profile-acts all the time, whereas in reality convective transport only occurs sporadically when the stratification becomes unstable. As a result, the dynamical vertical heat transport is much larger when using an unstable than a neutral radiative equilibrium profile in the Newtonian model.

We finally note a few other interesting aspects of our simulations. The tropopause structure in our simulations displays a jump from a high to a low tropopause as we move from the warm to the cool side of the jet, even though our model is dry and its tropical circulation shallow. In fact our "tropical tropopause" penetrates deeper than convection; see Fig. 2a. This seems consistent with recent modeling results by Haqq-Misra et al. (2011), who argue that the observed subtropical tropopause break in the atmosphere is produced by the eddies and does not require moisture. The formation of the tropopause break is probably best understood in terms of potential vorticity mixing by the eddies (Haynes et al. 2001). Since the jet maximum represents a barrier to PV mixing, large horizontal PV gradients develop there, with the result that PV contours become aligned quasi vertically over that region instead of horizontally as is typically the case away from the jet (see, e.g., the 1-PVU isoline in Fig. 5). The meridional wind curvature is important for the PV gradient at the jet location but as we move away from the jet to regions with negligible relative vorticity, the anomalously high (low) potential vorticity on the cool (warm) side of the jet must be associated with anomalously high (low) stratification. This 
is why the break in the thermal tropopause in our model tends to be located poleward of the jet maximum, though its precise location is sensitive to the lapse rate used in the definition of the thermal tropopause.

The aforementioned anomalous stratification is created by the residual vertical velocity, which cools (warms) the UTLS region on the warm (cool) side of the jet, raising the thermal tropopause over the former and lowering it over the latter, much as discussed by Thuburn and Craig (2000) and Birner (2010). The mixing barrier at the jet maximum plays an important role for this heating pattern by shaping the topology of the residual circulation. This is illustrated in Fig. 11, which compares schematically the residual mass flux in circulations with and without a mixing barrier. The first case corresponds to the Charney-like profile (unbounded jet) discussed in $\mathrm{ZGV}$. In the absence of a wind maximum there is no mixing barrier: rather, the eddy PV flux (and hence the residual meridional velocity $\bar{v}^{*} \sim-\overline{v^{\prime} q^{\prime}}(f)$ decreases uniformly with height as indicated by the increasing spacing of the residual streamlines. No tropopause break is found in this case. In contrast, in the presence of a zonal wind maximum-mixing barrier, the eddy PV flux only weakens locally and wave absorption recovers aloft (cf. Fig. 3f). This implies that the residual streamlines must divert around the wind maximum (middle panel of Fig. 11) as the circulation closes above the barrier, leading to a dipolar adiabatic heating pattern and to the formation of a thermal tropopause break. The full 2D picture is a bit more complicated. Instead of a broad meridional region with vanishing PV flux we observe a dipolar PV flux pattern, so that the meridional mean PV flux only vanishes because of cancellations when averaging (Fig. 4). The implication is that the residual meridional velocity also only vanishes in the meridional average, as residual trajectories retract back over the region with upgradient PV flux (bottom panel of Fig. 11). Thus, the descending flow is actually strongest over the barrier rather than poleward of it, which is consistent with the observed downward residual flow at the edge of the Hadley cell. This fine residual circulation structure is in good qualitative agreement with observations, in which a shallow reverse cell is also found on the poleward side of the subtropical jet [cf. Fig. 1 in Birner (2010)]. This reverse cell is already apparent in the pioneering study of Edmon et al. (1980) (see their Fig. 6a), though to the best of our knowledge this feature has received little attention in the literature. The residual circulation just described above, forced by the penetration of the tropospheric eddies above the region of positive EKE generation, is reminiscent of the shallow component of the Brewer-Dobson circulation in the real atmosphere (Plumb 2002). In contrast, the
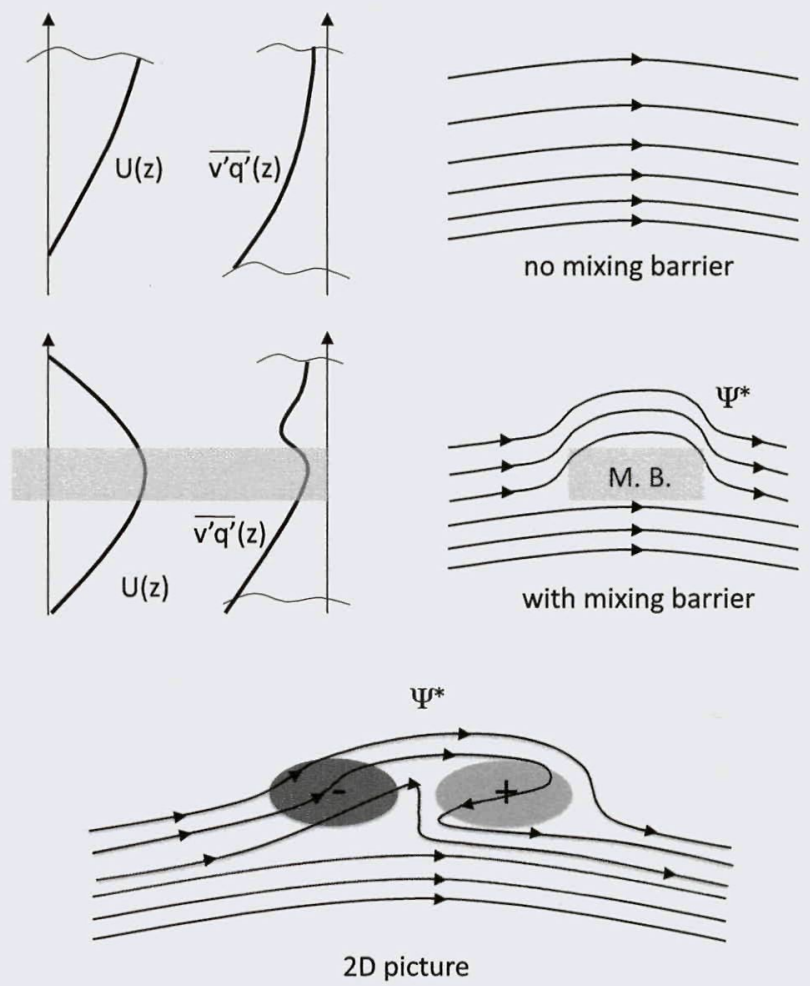

FIG. 11. Schematic illustrating the vertical structure of zonal wind and eddy PV flux and the topology of the residual streamlines: (top) 1D picture with an unbounded jet and no mixing barrier; (middle) as in (top), but with a jet stream maximum and a collocated mixing barrier; and (bottom) 2D picture in the presence of a dipolar PV flux pattern.

observed deep stratospheric circulation is forced by stratospheric eddies and thus absent in our simple model.

There are some similarities between the dynamics described in this paper and the arguments of Robinson (2006) for the self-maintenance of midlatitude jets. Robinson argues that an eddy-driven jet is self-maintained (in the sense that the net effect of the eddies is to reinforce the temperature gradient responsible for their generation) when the upper-level PV absorption has a local minimum over the baroclinic zone. Then, the temperature gradient is locally sharpened by the residual circulation over the generation region and smoothed on the sides, as the eddies propagate away from that region. Robinson shows that whether a jet is self-maintained or not is strongly linked to the formation of an extratropical upper-level wind maximum distinct from the subtropical jet, which, in the context of this paper, we might associate with the formation of a mixing barrier over the generation region. Interestingly, Robinson's arguments apply here not only in a meridional sense, but also vertically. In particular, the penetration of the tropospheric eddies 
above the mixing barrier into the region of EKE destruction also produces a dipolar PV flux pattern in the vertical direction in our model (Fig. 4). This pattern is associated with the weakening/reversal of the residual circulation at the jet stream level and its strengthening aloft. The enhanced vertical residual flow above the barrier leads to the reversal of the temperature gradient and the vertical sharpening of the jet as in Robinson's conceptual model. Also in agreement with Robinson (2006), this sharpening mechanism requires that the eddies are sufficiently strong. When $\Delta S$ is reduced in our model, the upper-level wind maximum broadens vertically (not shown) and eventually disappears when baroclinicity is sufficiently weak (see line with square markers in Fig. 8a).

We end with a caveat. In spite of using a gray radiation scheme, our model is not realistic in many respects. In an attempt to model a semi-infinite fluid in a way that does not (for simplicity) directly involve a density scale height we have used a Boussinesq fluid. However, we still use a cooling temperature, related to the real temperature through a fictitious scale height $\left(H_{c}\right)$, to calculate infrared emission in our gray radiation model (otherwise the model would convect to the top). Interestingly, this scale, which we may interpret as the depth over which infrared emission is efficient in cooling off the atmosphere, was found to be an important parameter controlling tropopause height in our model. We expect that the same should be true in the real, compressible atmosphere.

Acknowledgments. P. Z.-G. acknowledges support from the MOVAC (Grant 200800050084028 from the Ministerio de Medio Ambiente, y Medio Rural y Marino of Spain), DEVIAJE (CGL2009-06944), and COMETH (CGL2012-30641) projects. G.K.V. was funded by the National Science Foundation under Grant AGS-1144302. We are grateful to Peter Haynes and the anonymous reviewers for their insightful comments, which greatly improved the scientific depth of our manuscript. We credit a reviewer with pointing out the relation between our work and that of Robinson.

\section{REFERENCES}

Andrews, D., J. Holton, and C. Leovy, 1987: Middle Atmosphere Dynamics. Academic Press, 498 pp.

Birner, T., 2006: Fine-scale structure of the extratropical tropopause region. J. Geophys. Res., 111, D04104, doi:10.1029/ 2005JD006301.

, 2010: Residual circulation and tropopause structure. J. Atmos. Sci., 67, 2582-2600.

- and D. W. Thompson, 2011: Counter-gradient eddy fluxes of potential vorticity near the subtropical jet. Extended Abstracts, 18th Conf. on Atmospheric and Oceanic Fluid Dynamics, Spokane, WA, Amer. Meteor. Soc., 16.7.

Edmon, H. J., B. J. Hoskins, and M. E. McIntyre, 1980: EliassenPalm cross sections for the troposphere. J. Atmos. Sci., 37, 2600-2616.

Frierson, D. M. W., I. M. Held, and P. Zurita-Gotor, 2006: A gray radiation aquaplanet moist GCM. Part I: Determination of the atmospheric static stability and eddy scale. J. Atmos. Sci., 63, 2548-2566.

Fueglistaler, S., A. E. Dessler, T. J. Dunkerton, I. Folkins, Q. Fu, and P. W. Mote, 2009: Tropical tropopause layer. Rev. Geophys., 47, RG1004, doi:10.1029/2008RG000267.

Gettelman, A., P. Hoor, L. L. Pan, W. J. Randel, M. I. Hegglin, and T. Birner, 2011: The extratropical upper troposphere and lower stratosphere. Rev. Geophys., 49, RG3003, doi:10.1029/ 2011RG000355.

Greenslade, M., and P. Haynes, 2008: Vertical transition in transport and mixing in baroclinic flows. J. Atmos. Sci., 65, 1137-1157.

Haqq-Misra, J., S.-Y. Lee, and D. Frierson, 2011: Tropopause structure and the role of eddies. J. Atmos. Sci., 68, 2930-2944.

Haynes, P., J. Scinocca, and M. Greenslade, 2001: Formation and maintenance of the extratropical tropopause by baroclinic eddies. Geophys. Res. Lett., 28, 4179-4182.

Held, I. M., 1978: The vertical scale of an unstable baroclinic wave and its importance for eddy heat flux parameterizations. J. Atmos. Sci., 35, 572-576.

- 1982: On the height of the tropopause and the static stability of the troposphere. J. Atmos. Sci., 39, 412-417.

- , and T. Schneider, 1999: The surface branch of the zonally averaged mass transport circulation in the troposphere. J. Atmos. Sci., 56, 1688-1697.

Hoerling, M., T. Schaack, and A. Lenzen, 1991: Global objective tropopause analysis. Mon. Wea. Rev., 119, 1816-1832.

Jansen, M., and R. Ferrari, 2012: Macroturbulent equilibration in a thermally forced primitive equation system. J. Atmos. Sci., 69, 695-713.

Kunz, A., P. Konopka, R. Müller, and L. L. Pan, 2011: Dynamical tropopause based on isentropic potential vorticity gradients. J. Geophys. Res., 116, D01110, doi:10.1029/2010JD014343.

Marshall, J., A. Adcroft, C. Hill, L. Perelman, and C. Heisey, 1997: A finite-volume, incompressible Navier Stokes model for studies of the ocean on parallel computers. J. Geophys. Res., 102 (C3), 5753-5766.

Pan, L. L., W. J. Randel, B. L. Gary, M. J. Mahoney, and E. J. Hintsa, 2004: Definitions and sharpness of the extratropical tropopause: A trace gas perspective. J. Geophys. Res., 109, D23103, doi:10.1029/2004JD004982.

Plumb, R. A., 2002: Stratospheric transport. J. Meteor. Soc. Japan, 80, 793-809.

Robinson, W. A., 2006: On the self-maintenance of midlatitude jets. J. Atmos. Sci., 63, 2109-2122.

Schneider, T., 2004: The tropopause and thermal stratification in the extratropics of a dry atmosphere. J. Atmos. Sci., 61, 13171340.

—, and C. C. Walker, 2006: Self-organization of atmospheric macroturbulence into critical states of weak nonlinear eddyeddy interactions. J. Atmos. Sci, 63, 1569-1586.

Stone, P. H., 1978: Baroclinic adjustment. J. Atmos. Sci., 35, 561571.

Thuburn, J., and G. C. Craig, 1997: GCM tests of theories for the height of the tropopause. J. Atmos. Sci., 54, 869-882.

-, and _ 2000: Stratospheric influence on tropopause height: The radiative constraint. J. Atmos. Sci, 57, 17-28. 
World Meteorological Organization, 1957: Definition of the tropopause. WMO Bull., 6, 136.

Zurita-Gotor, P., 2008: The sensitivity of the isentropic slope in a primitive equation dry model. J. Atmos. Sci., 65, 43-65.

- , and R. S. Lindzen, 2007: Theories of baroclinic adjustment and eddy equilibration. The Global Circulation of the Atmosphere, T. Schneider and A. H. Sobel, Eds., Princeton University Press, 22-46.
, and G. K. Vallis, 2009: Equilibration of baroclinic turbulence in primitive-equation and quasi-geostrophic models. J. Atmos. Sci., 66, 837-863.

- and - 2010: Circulation sensitivity to heating in a simple model of baroclinic turbulence. J. Atmos. Sci., 67, 15431558.

—, and - 2011: Dynamics of midlatitude tropopause height in an idealized model. J. Atmos. Sci., 68, 823-838. 